\title{
Article
}

\section{On the existence of solutions for fractional boundary valued problems with integral boundary conditions involving measure of non compactness}

\author{
Ahmed Hamrouni ${ }^{1}$ and Said Beloul ${ }^{1, *}$ \\ 1 Department of Mathematics, Exact Sciences Faculty, University of El Oued, P.O.Box 789, El Oued 39000, Algeria. \\ * Correspondence: beloulsaid@gmail.com
}

Received: 15 June 2020; Accepted: 30 July 2020; Published: 31 August 2020.

\begin{abstract}
This paper presents an existence theorem of the solutions for a boundary value problem of fractional order differential equations with integral boundary conditions, by using measure of noncompactness combined with Mönch fixed point theorem. An example is furnished to illustrate the validity of our outcomes.
\end{abstract}

Keywords: Fixed point, measure of noncompactness, fractional differential equation.

MSC: 34A08, 34B15, 34G20, 47H08.

\section{Introduction}

Q uite recently, fractional differential equations become one of the most important research topic, since their applications in various applied science, as in physics, finance, hydrology, biophysics, thermodynamics, control theory, statistical mechanics, for example see [1,2]. many results were given concerning the existence and uniqueness of the solution of such equations by using various techniques, while the fixed point theory tool still one of the efficacy methods, see for example. Several authors tried to develop a technique that depends on the Darbo or the Monch fixed point theorems with the Hausdorff or Kuratowski measure of noncompactness.

This article deals with the existence of solutions for boundary value problems with fractional order differential equations and nonlinear integral boundary conditions. We furnish an example to illustrate our results.

Consider the following boundary value problems:

$$
\left\{\begin{array}{l}
{ }^{c} D^{\alpha} x(t)+f\left(t, x(t),{ }^{c} D^{\alpha} x(t)\right)=0,0 \leq t \leq 1,1<\alpha \leq 2 \\
a x(0)-b x^{\prime}(0)=0 \\
x(1)=\int_{0}^{1} g(s, x(s)) d s+\lambda
\end{array}\right.
$$

where $\lambda>0,{ }^{c} D^{\alpha}, 1<\alpha \leq 2$ is the Caputo fractional derivative, $f$ and $g$ are given functions

$f:[0 ; 1] \times C([0 ; 1] ; \mathbb{R}) \times \mathbb{R} \longrightarrow R, g \in C([0,1], \mathbb{R}), a, b, \lambda \in \mathbb{R}_{+}, a+b>0$ and $\frac{a}{a+b}<\alpha-1$.

\section{Preliminaries}

Now, we give some definitions and preliminaries which will be used throughout this paper. Let $X=$ $C([0,1], \mathbb{R})$ be the Banach space of continuous functions $x:[0,1] \longrightarrow \mathbb{R}$, with the usual supermum norm

$$
\|x\|_{\infty}=\sup \{\|x(t)\|, \quad t \in[0,1]\} .
$$


Let $L^{1}([0 ; 1], \mathbb{R})$ be the Banach space of measurable functions $x:[0,1] \longrightarrow \mathbb{R}$ which are Bochner integrable, equipped with the norm

$$
\|x\|_{L^{1}}=\int_{0}^{1} x(t) d t .
$$

Now let us recall some fundamental facts of the notion of Kuratowski measure of noncompactness.

Definition 1. [3,4] Let $E$ be a Banach space and $\Omega_{E}$ be the bounded subsets of $E$. The Kuratowski measure of noncompactness is the map $\alpha: \Omega_{E} \rightarrow[0, \infty]$ defined by

$$
\mu(B)=\liminf \left\{\epsilon>0: B \subseteq \cup_{i=1}^{n} B_{i}, \operatorname{diam}\left(B_{i}\right) \leq \epsilon\right\}, B \in \Omega_{E},
$$

where

$$
\operatorname{diam}\left(B_{i}\right)=\sup \left\{\|u-v\|_{E}: u, v \in B_{i}\right\}
$$

The Kuratowski measure of noncompactness satisfies the following properties;

Lemma 1. [3,4] Let $A$ and $B$ bounded sets. Then

1. $\mu(B)=0$ if and only if $\bar{B}$ is compact ( $B$ is relatively compact).

2. $\mu(B)=\mu(\bar{B})$.

3. $A \subset B \Rightarrow \mu(A) \leq \mu(B)$.

4. $\mu(A+B) \leq \mu(A)+\mu(B)$.

5. $\mu(c B) \leq|c| \mu(B), c \in \mathbb{R}$.

6. $\mu(\operatorname{conv} B)=\mu(B)$.

Definition 2. [5] The Riemann Liouville fractional integral of order $q>0$ of $x:(0, \infty) \rightarrow \mathbb{R}$ is given by

$$
I_{0^{+}}^{\alpha} x(t)=\frac{1}{\Gamma(\alpha)} \int_{0}^{t}(t-s)^{\alpha-1} x(s) d s
$$

provided that the right hand side is defined on $(0, \infty)$.

Definition 3. [5] The Riemann Liouville fractional derivative of order $q \in(0,1) 0$ of $x:(0, \infty) \rightarrow \mathbb{R}$ is given by

$$
{ }^{R L} D_{0^{+}}^{\alpha} x(t)=\frac{1}{\Gamma(n-q)} \frac{d^{(n)}}{d t} \int_{0}^{t}(t-s)^{n-q-1} x(s) d s, \quad n=[q]+1,
$$

where $\Gamma(q)$ denotes the classical gamma function, provided that the right-hand side is pointwise defined on $(0, \infty)$.

Definition 4. [5] The fractional derivative in Caputo sense of order $q>0$ for a function $x:(0, \infty) \rightarrow \mathbb{R}$ is given by

$$
{ }^{c} D^{\alpha} x(t)=\frac{1}{\Gamma(n-q)} \int_{0}^{t}(t-s)^{n-q-1} x^{(n)}(s) d s,
$$

where $n=[q]+1$, provided that the right side is pointwise defined on $(0, \infty)$.

Lemma 2. [5] Let $\alpha>0$, then the differential equation

$$
{ }^{c} D^{\alpha} x(t)=0
$$

has solutions $x(t)=\sum_{i=0}^{n-1} c_{i} t^{i}, c_{i} \in \mathbb{R}, n=[\alpha]+1$.

Lemma 3. [5] Let $\alpha>0$, then

$$
I^{\alpha c} D^{\alpha} x(t)=x(t)-\sum_{i=0}^{n-1} c_{i} t^{i}, c_{i} \in \mathbb{R}, n=[\alpha]+1
$$


Theorem 1. [1] Let $X$ be a Banach space and $0 \in C$ be a nonempty, bounded, closed and convex subset of X. Suppose a continuous mapping $N: C \rightarrow C$ is such that for all non empty subsets $V$ of $C$,

$$
\mu(N(V)) \leq k \mu(V)
$$

where $0 \leq k<1$, and $\mu$ is the Kuratowski measure of noncompactness, then $N$ has a fixed point in $C$.

Theorem 2. [6] Let $C$ be a bounded, closed and convex subset of a Banach space such that $0 \in C$, and let $T$ be a continuous mapping of $C$ into itself. If the implication

$$
V=\overline{\operatorname{conv}} T(V) \text { or } V=T(V) \cup 0 \Rightarrow \mu(V)=0
$$

holds for every subset $V$ of $C$, then $T$ has a fixed point.

Lemma 4. [7] Let $D$ be a bounded, closed and convex subset of the Banach space $C(J, X), G$ a continuous function on $J \times J$ and $f$ a function from $J \times X \rightarrow X$ which satisfies the Carathéodory conditions, and suppose there exists $p \in L^{1}\left(J, R_{+}\right)$such that, for each $t \in J$ and each bounded set $B \subset X$, we have

$$
\lim _{h \rightarrow 0^{+}} \mu\left(f\left(J_{t, h} \times B\right)\right) \leq p(t) \mu(B) ; \text { here } J_{t, h}=[t-h, t] \cap J .
$$

If $V$ is an equicontinuous subset of $D$, then

$$
\mu\left(\left\{\int_{J} G(s, t) f(s, x(s)) d s: x \in V\right\}\right) \leq \int_{J}\|G(t, s)\| p(s) \mu(V(s)) d s .
$$

Lemma 5. Let $X$ be a Banach space and $F \subset C(J, X)$. If the following conditions are satisfied:

- family $F$ in $C(J, X)$ is called uniformly bounded if there exists a positive constant $K$ such that $|f(t)| \leq K$ for all $t \in J$ and all $f \in F$;

- $F$ is called equicontinuous, if for every $\epsilon>0$ there exists a $\delta>0$ such that $\left|f\left(t_{1}\right) \cdot f\left(t_{2}\right)\right|<\varepsilon$ for all $t_{1}, t_{2} \in J$ with $\left|t_{1}-t_{2}\right|<\delta$ and all $f \in F$

- for each $x \in E, A(x)=\{f(x) ; f \in A\}$ is relatively compact.

\section{Main results}

Lemma 6. Let $1<\alpha<2$ and $y \in C([0,1])$. A function $x$ is a solution of the fractional integral equation

$$
x(t)=\int_{0}^{1} G(t, s) f(s) d s+\frac{a t+b}{a+b} \int_{0}^{1} g(s) d s+\frac{a t+b}{a+b} \lambda,
$$

where $G$ is the Green function given by

$$
G(t, s)= \begin{cases}\frac{(a t+b)(1-s)^{\alpha-1}}{(a+b) \Gamma(\alpha)}-\frac{(t-s)^{\alpha-1}}{\Gamma(\alpha)} & s \leq t \\ \frac{(a t+b)(1-s)^{\alpha-1}}{(a+b) \Gamma(\alpha)} & t \leq s\end{cases}
$$

if and only if $x$ is a solution of the fractional boundary value problem

$$
\left\{\begin{array}{l}
{ }^{c} D^{\alpha} x(t)=-f(t), 0 \leq t \leq 1,1<\alpha \leq 2 \\
a x(0)-b x^{\prime}(0)=0 \\
x(1)=\int_{0}^{1} g(s) d s+\lambda
\end{array}\right.
$$

Proof. By Lemma 3, we reduce (4)) to an equivalent integral equation

$$
\begin{aligned}
& x(t)=-\frac{1}{\Gamma(\alpha)} \int_{0}^{t}(t-s)^{\alpha-1} f(s) d s+c_{0}+c_{1} t \\
& x^{\prime}(t)=-\frac{1}{\Gamma(\alpha-1)} \int_{0}^{t}(t-s)^{\alpha-2} f(s) d s+c_{1}
\end{aligned}
$$


for some constants $c_{0}, c_{1} \in X$. Boundary conditions of (4) give

$$
\begin{gathered}
a c_{0}-b c_{1}=0 \\
-\frac{1}{\Gamma(\alpha)} \int_{0}^{1}(1-s)^{\alpha-1} f(s) d s+c_{0}+c_{1}=\int_{0}^{1} g(s) d s+\lambda .
\end{gathered}
$$

Therefore

$$
\left\{\begin{array}{l}
c_{0}=\frac{b}{a+b}\left[\frac{1}{\Gamma(\alpha)} \int_{0}^{1}(1-s)^{\alpha-1} f(s) d s+\int_{0}^{1} g(s) d s+\lambda\right], \\
c_{1}=\frac{a}{a+b}\left[\frac{1}{\Gamma(\alpha)} \int_{0}^{1}(1-s)^{\alpha-1} f(s) d s+\int_{0}^{1} f(s) d s+\lambda\right] .
\end{array}\right.
$$

Thus

$$
\begin{aligned}
x(t)= & -\frac{1}{\Gamma(\alpha)} \int_{0}^{t}(t-s)^{\alpha-1} f(s) d s+\frac{b}{a+b}\left[\frac{1}{\Gamma(\alpha)} \int_{0}^{1}(1-s)^{\alpha-1} f(s) d s+\int_{0}^{1} h(s) y(s) d s+\lambda\right] \\
& +\frac{a t}{a+b}\left[\frac{1}{\Gamma(\alpha)} \int_{0}^{1}(1-s)^{\alpha-1} k(s) d s+\int_{0}^{1} g(s) d s+\lambda\right] \\
& \times \int_{0}^{t}\left[\frac{(a t+b)(1-s)^{\alpha-1}}{(a+b) \Gamma(\alpha)}-\frac{(t-s)^{\alpha-1}}{\Gamma(\alpha)}\right] f(s) d s+\int_{t}^{1} \frac{(a t+b)(1-s)^{\alpha-1}}{(a+b) \Gamma(\alpha)} f(s) d s \\
& +\frac{a t+b}{a+b} \int_{0}^{1} g(s) d s+\frac{a t+b}{a+b} \lambda \\
= & \int_{0}^{1} G(t, s) f(s) d s+\frac{a t+b}{a+b} \int_{0}^{1} g(s) d s+\frac{a t+b}{a+b} \lambda .
\end{aligned}
$$

The proof is complete.

Since, we have

$$
\begin{aligned}
\int_{0}^{1} G(t, s) d s= & \frac{1}{\Gamma(\alpha)}\left(\int 0^{t}(t-s)^{\alpha-1} d s+\frac{a t+b}{a+b} \int 0^{1}(1-s)^{\alpha-1} d s\right) \\
& \frac{1}{\Gamma(\alpha+1)}\left(t^{\alpha}+\frac{a t+b}{a+b}\right) \leq \frac{2}{\Gamma(\alpha+1)} .
\end{aligned}
$$

Assume that

(A1): There exist $K>0$ and $L>0$ such that

$$
|f(t, x(t), u(t))-f(t, y(t), v(t))| \leq K\|x-y\|_{\infty}+L\|u-v\|_{\infty} .
$$

(A2): For any bounded subset $A$ and $B$ of $X$ we have

$$
\mu(f(t, A, B)) \leq K \mu(A)+L \mu(B),
$$

or $\mu$ is a measure of non-compactness.

(A3): There exists $N>0$ such that

$$
\| g(t, x(t))-g(t, y(t)\|\leq N\| x-y \| .
$$

(A4): For any bounded subset $C$ of $X$, we have

$$
\mu(g(t, C)) \leq N \mu(C) .
$$

(A5):

$\frac{K}{(1-L) \mu(\alpha+1)}+N<1$

Theorem 3. Under the assumptions (A1)-(A5) the Problem (1) has a solution provided

$$
\frac{2 K}{(1-L) \Gamma(\alpha+1)}+N<1 .
$$


Proof. Transform the Problem (1) into a fixed point problem. Consider the operator $T: X=C([0,1], \mathbb{R}) \longrightarrow$ $C([0,1], \mathbb{R})$ defined by

$$
T x(t)=\int_{0}^{1} G(t, s) f\left(t, x(s),{ }^{c} D^{\alpha} x(s)\right) d s+\frac{a t+b}{a+b} \int_{0}^{1} g(s, x(s)) d s+\frac{a t+b}{a+b} \lambda .
$$

Clearly, the fixed points of the operator $T$ are solutions of the Problem (1). We shall show that $T$ satisfies the assumptions of Theorem 3. The proof will be given in three steps.

Step 1: $T$ is continuous. Let $\left(x_{n}\right)$ be a sequence such that $x_{n} \longrightarrow x$ in $C([0,1], X)$, then for each $t \in[0,1]$ we have

$$
\begin{aligned}
\left|T\left(x_{n}\right)(t)-T(x)(t)\right| \leq & \left|\int_{0}^{1}\right| G(t, s) f\left(t, x_{n}(t),{ }^{c} D^{\alpha} x_{n}(t)\right)-f\left(t, x(t),{ }^{c} D^{\alpha} x(t) \mid d s\right. \\
& +\frac{a t+b}{a+b} \int_{0}^{1}\left(g\left(s, x_{n}(s)\right)-g(s, x(s)) \mid d s .\right.
\end{aligned}
$$

By $(A 1)$ and $(A 3)$, we have

$$
\begin{aligned}
\mid f\left(t, x_{n}(t),{ }^{c} D^{\alpha} x_{n}(t)\right)-f\left(t, x(t),{ }^{c} D^{\alpha} x(t) \mid\right. & \leq K\left|x_{n}-x\right|+L\left|{ }^{c} D^{\alpha} x_{n}(t)-{ }^{c} D^{\alpha} x(t)\right| \\
& \leq K\left|x_{n}-x\right|+L\left|f\left(t, x_{x}(t),{ }^{c} D^{\alpha} x_{n}(t)\right)-f\left(t, x(t),{ }^{c} D^{\alpha} x(t)\right)\right| .
\end{aligned}
$$

Then

$$
\mid f\left(t, x_{n}(t),{ }^{c} D^{\alpha} x_{n}(t)\right)-f\left(t, x(t),{ }^{c} D^{\alpha} x(t)\left|\leq \frac{K}{1-L}\right| x_{n}-x \mid,\right.
$$

and

$$
\left|g_{t}, x_{n}(t)-g(t, x(t))\right| \leq N\left|x_{n}-x\right|
$$

So

$$
\left|T\left(x_{n}\right)(t)-T(x)(t)\right| \leq\left(\frac{2 K}{1-L}+N\right) \| x_{n}-x \mid .
$$

Since $x_{n} \longrightarrow x$, for each $t \in[0,1]$.

$$
\left|T\left(x_{n}(t)-T(x)(t)\right)\right| \longrightarrow 0, \quad \text { when } n \longrightarrow \infty .
$$

So

$$
\left\|T\left(x_{n}\right)(t)-T(x)(t)\right\| \rightarrow 0, \text { when } n \rightarrow \infty .
$$

Therefore, $T$ is continuous. Now, let

$$
r \geq \frac{1}{(1-L) \Gamma(\alpha+1)}\left(K r+f_{0}\right)+N r+g_{0}+\lambda
$$

where

$$
f_{0}=\sup _{t \in[0,1]}\|f(t, 0,0)\|, \quad g_{0}=\sup _{t \in[0,1]}\|g(t, 0)\| .
$$

Define $B_{r}=\{x \in X:\|x\| \leq r\}$. It is clear that $B_{r}$ is a bounded, closed and convex subset of $X$.

Step 2: $T$ maps $B_{r}$ into itself, i.e., $T\left(B_{r}\right) \subseteq B_{r}$. Let $x \in B_{r}$, so for each $t \in[0,1]$, we have

$$
\begin{aligned}
|T(x)(t)| & =\left|\int_{0}^{1} G(t, s) f\left(t, x_{n}(t),{ }^{c} D^{\alpha} x(t)\right) d s+\frac{a t+b}{a+b} \int_{0}^{1} g(s, x(s)) d s+\frac{a t+b}{a+b} \lambda\right| \\
& \leq \int_{0}^{1} G(t, s) f\left(t, x_{n}(t),{ }^{c} D^{\alpha} x_{n}(t)\right) d s+\frac{a t+b}{a+b} \int_{0}^{1} \int_{0}^{1} g(s, x(s)) d s+\frac{a t+b}{a+b} \lambda \\
& \leq \frac{2}{\Gamma(\alpha+1)}\left\|f\left(s, x(s),{ }^{c} D^{\alpha} x(t)\right)\right\|+\|g(s, x(s))\|+\lambda .
\end{aligned}
$$

By $(A 1)$ et $(A 3)$, we have for each $t \in[0,1]$ 


$$
\begin{aligned}
\left\|f\left(s, x(s),{ }^{c} D^{\alpha} x(t)\right)\right\| & =\left\|f\left(t, x(t),{ }^{c} D^{\alpha} x(t)\right)-f(t, 0,0)+f(t, 0,0)\right\| \\
& \leq\left\|f\left(t, x,{ }^{c} D^{\alpha} x(t)\right)-f(t, 0,0)\right\|+\|f(t, 0,0)\| \\
& \leq K\|x\|+L\left\|^{c} D^{\alpha} x(t)\right\|+f_{0} \\
& \leq \frac{1}{1-L}\left(K r+f_{0}\right)
\end{aligned}
$$

and

$$
\begin{aligned}
\|g(t, x(t))\| & =\|g(t, x(t))-g(t, 0)+g(t, 0)\| \\
& \leq\|g(t, x(t))-g(t, 0)\|+\|g(t, 0)\| \\
& \leq N\|x\|+g_{0} \\
& \leq N r+g_{0} .
\end{aligned}
$$

So

$$
\left.\|T x(t)\| \leq \frac{2}{(1-L) \Gamma(\alpha+1)}\left(K r+f_{0}\right)+N r+g_{0}\right)+\lambda \leq r .
$$

It follows that for each $t \in[0,1]$, we have

$$
\|T x(t)\| \leq r \text { which implies } T\left(B_{r}\right) \subseteq B_{r} .
$$

Step 3: $T\left(B_{r}\right)$ is bounded and equicontinuous. According to step 2, we have

$$
T\left(B_{r}\right)=\left\{T(x): x \in B_{r}\right\} \subset B_{r} .
$$

So for every $x \in B_{r}$ we have $\|T(x)\|_{\infty} \leq r$, which means that $T\left(B_{r}\right)$ is bounded. Now, let $t_{1}, t_{2} \in[0,1], t_{1}<$ $t_{2}$ and $x \in T\left(B_{r}\right)$, then

$$
\left|T(x)\left(t_{2}\right)-T(x)\left(t_{1}\right)\right| \leq\left\|f\left(s, x(s),{ }^{c} D^{\alpha} x(t)\right)\right\| \int_{0}^{1}\left|G\left(t_{2}, s\right)-G\left(t_{1}, s\right)\right| d s+\frac{a\left|t_{2}-t_{1}\right|}{a+b}(\|g(t, x(t))\|+\lambda) .
$$

According to (5) and (6), we have

$$
\left\|T(x)\left(t_{2}\right)-T(x)\left(t_{1}\right)\right\| \leq \frac{1}{1-L}\left(K r+f_{0}\right)\left|t_{2}^{\alpha}-t_{1}^{\alpha}\right|+\frac{a\left|t_{2}-t_{1}\right|}{a+b}\left(N r+g_{0}+\lambda\right) .
$$

As $t_{2} \longrightarrow t_{1}$, the right hand side of the above inequality tends to zero, so $T$ is equicontinuous. Let $V \subset T B_{r}$, such as $V=\left\{T x, x \in B_{r}\right\}$, so $V \subset \overline{\operatorname{conv}}(T(V) \cup\{0\})$. The subset $V$ is bounded and equicontinuous, so the function $t: \longmapsto \mu(V(t)) \in \mathbb{R}$ is continuous on $[0,1]$. Using Lemma 4 and the properties of the measure $\mu$, we have, for each $t \in[0,1]$

$$
\begin{aligned}
v(t)=\mu(V(t)) & \leqslant \mu(T(V)(t) \cup\{0\}) \\
& \leqslant \mu(T(V)(t)) \\
& =\mu\left(\left\{\int_{0}^{1} G(t, s) f\left(t, x(t),{ }^{c} D^{\alpha} x(t)\right) d s+\frac{a t+b}{a+b} \int_{0}^{1} g(s, x(s)) d s+\frac{a t+b}{a+b} \lambda, x \in V\right\}\right) \\
& \leq \mu\left(\left\{\int_{0}^{1} G(t, s) f\left(t, x(t),{ }^{c} D^{\alpha} x(t)\right) d s, x \in V\right\}\right)+\mu\left(\left\{\frac{a t+b}{a+b}\left(\int_{0}^{1} g(s, x(s)) d s+\lambda, x \in V\right\}\right)\right. \\
& \leq \int_{0}^{1} G(t, s) \mu\left(f\left(t, x(t),{ }^{c} D^{\alpha} x(t)\right)\right) d s+\frac{a t+b}{a+b} \int_{0}^{1} \mu(g(s, x(s))) d s .
\end{aligned}
$$

Thus for each $s \in[0,1]$,

$$
\begin{aligned}
\mu\left(\left\{f\left(s, x(t),{ }^{c} D^{\alpha} x(t)\right), x(i) \in V\right\}\right) & \leq K \mu(\{x(t), x \in V\})+L \mu\left(\left\{f\left(s, x(t),{ }^{c} D^{\alpha} x(t)\right)\right\}\right) \\
& \leq \frac{K}{1-L} \mu(\{x(s), x \in V\})=\frac{K}{1-L} v(t),
\end{aligned}
$$


and

$$
\mu(g(t, V(t))=\mu(\{g(t, x(t), x(t) \in V(t)\}) \leq N v(t) .
$$

Then

$$
\begin{aligned}
\mu(T(V)(t)) \leq & \frac{2 K}{(1-L) \Gamma(\alpha+1)} \mu(V(t))+\frac{a t+b}{a+b} N \mu(V(t)) \\
& \leq \frac{2 K}{(1-L) \Gamma(\alpha+1)} v(t)++N v(t) \\
& =\left(\frac{2 K}{(1-L) \Gamma(\alpha+1)}+N\right)\|v\|_{\infty} .
\end{aligned}
$$

So

$$
\|v\|_{\infty} \leq\left(\frac{2 K}{(1-L) \Gamma(\alpha+1)}+N\right)\|v\|_{\infty}
$$

which gives $\|v\|_{\infty}=0$, that is to say $v(t)=0$, for each $t \in[0,1]$, then $V(t)$ is relatively compact in $X$. In view of the lemma of Ascoli - Arzela, $V$ is relatively compact in $B_{r}$. Applying now the Theorem of Mönch, we conclude that $T$ has a fixed point which is a solution of the Problem (1). This completes the proof.

Finally, we present a numerical example to illustrate our results.

Example 1. Let us consider the following fractional boundary value problem:

$$
\left\{\begin{array}{l}
{ }^{c} D^{\frac{3}{2}} x(t)=\frac{1}{10}\left(t \cos x-x e^{t}\right)+\frac{1}{9} \cos \left({ }^{c} D^{\frac{3}{2}} x(t)\right), t \in[0,1] \\
x(0)-x^{\prime}(0)=0 \\
x(1)=\frac{1}{8} \int_{0}^{1} s e^{-s} x(s) d s
\end{array}\right.
$$

where

$$
f(t, x, u)=\frac{1}{10}\left(t \cos (x)-x e^{t}\right)+\frac{1}{9} \cos \left({ }^{c} D^{\frac{3}{2}} x(t)\right), x, u \in C([0,1], \mathbb{R})
$$

and

$$
g(s, x)=\frac{x(s)}{8}, \quad g \in C(I \times \mathbb{R}, \mathbb{R}), \alpha=\frac{3}{2}, a=1, b=1 .
$$

Clearly $\frac{a}{a+b}=\frac{1}{3}<\alpha-1$. On other hand we have

$\|f(t, x, u)-f(t, y, v)\| \leq \frac{1}{10}|t|\|\cos x-\cos y\|+\frac{1}{10}\left|e^{t}\right|\|x-y\|+\frac{1}{9}\|\cos u-\cos v\| \leq \frac{1+e}{10}\|x-y\|+\frac{1}{9}\|u-v\|$.

Therefore, the conditions $(A 1)$ and $(A 3)$ are satisfied with $K=\frac{1+e}{10}, L=\frac{1}{9}, N=\frac{1}{8}$ and

$$
\frac{K}{\Gamma(\alpha+1)(1-L)}+N=\frac{3(1+e)}{10 \sqrt{\pi}}+\frac{1}{8}<1 .
$$

Then the Problem (7) admits at least one solution on $[0,1]$.

Author Contributions: All authors contributed equally to the writing of this paper. All authors read and approved the final manuscript.

Conflicts of Interest: "The authors declare no conflict of interest."

\section{References}

[1] Darbo, G. (1955). Punti uniti in trasformazioni a codominio non compatto. Rendiconti del Seminario matematico della Università di Padova, 24, 84-92.

[2] Miller, K. S., \& Ross, B. (1993). An introduction to the fractional calculus and fractional differential equations. Wiley.

[3] Akhmerov, R. R., Kamenskii, M. I., Potapov, A. S., Rodkina, A. E., \& Sadovskii, B. N. (1992). Measures of noncompactness and condensing operators (Vol. 55). Basel: Birkhäuser. 
[4] Banas, J. (1980). On measures of noncompactness in Banach spaces. Commentationes Mathematicae Universitatis Carolinae, 21(1), 131-143.

[5] Kilbas, A. A., Srivastava, H. M., \& Trujillo, J. J. (2006). Theory and applications of fractional differential equations (Vol. 204). elsevier.

[6] Mönch, H. (1980). Boundary value problems for nonlinear ordinary differential equations of second order in Banach spaces. Nonlinear Analysis: Theory, Methods E Applications, 4(5), 985-999.

[7] Szufla, S. (1986). On the application of measure of noncompactness to existence theorems. Rendiconti del Seminario Matematico della Universita di Padova, 75, 1-14.

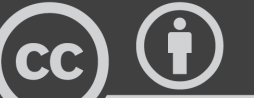

BY

(c) 2020 by the authors; licensee PSRP, Lahore, Pakistan. This article is an open access article distributed under the terms and conditions of the Creative Commons Attribution (CC-BY) license (http://creativecommons.org/licenses/by/4.0/). 